\title{
GAPcare: the Geriatric Acute and Post-acute Fall Prevention Intervention-a pilot investigation of an emergency department- based fall prevention program for community-dwelling older adults
}

\author{
Elizabeth M. Goldberg ${ }^{1,2^{*}}$ (D), Linda Resnik ${ }^{2,3}$, Sarah J. Marks ${ }^{4}$ and Roland C. Merchant ${ }^{1,4}$
}

\begin{abstract}
Background: Falls are the leading cause of fatal and non-fatal injuries among older adults. Older emergency department (ED) patients who present for evaluations after falls have a 30\% higher risk of falling again in the subsequent 6 months than age-matched controls. Although EDs frequently evaluate older adults after their falls, the typical evaluation consists of an injury assessment alone. As such, an opportunity is lost to assess and address the potential causes of falls in this vulnerable population. In this manuscript, we present a multidisciplinary fall prevention protocol for a pilot study of older adult ED patients who recently sustained a fall (GAPcare: the Geriatric Acute and Post-acute Fall Prevention Intervention).

Methods: GAPcare is a randomized single-blinded pilot study. Participants in GAPcare are 120 older adults ( $\geq 65$ years old) who present to 1 of 2 academic US EDs after a fall. We randomly assign participants 1:1 to an intervention or a usual care (control) arm. In the intervention arm, the patient's ED physician, a pharmacist, and a physical therapist (PT) collaborate to identify and address any risk factors that may have contributed to the fall. Intervention arm participants and their caregivers return home with a medication-related action plan to taper or stop potentially inappropriate medications and to address polypharmacy and a PT assessment and plan. Participants in the usual care arm receive standard assessments and care in the ED and a home safety brochure. Participants in both study arms complete fall calendars for 6 months to document the number of falls and healthcare visits during follow-up. The primary outcome is feasibility of the GAPcare fall prevention intervention (number and proportion of screened participants who are eligible, recruited, and retained; impact on ED length of stay), while the secondary outcome is to estimate its initial efficacy.
\end{abstract}

Discussion: The GAPcare-ED fall prevention intervention has the potential to promote older adult-sensitive care for millions of Americans presenting to EDs after falls and establish a protocol for a future large-scale randomized controlled trial on this topic.

Trial registration: ClinicalTrials.gov, NCT03360305. Trial registration date: December 4, 2017. Protocol version: 1

Keywords: Care transitions, Emergency department, Older adults, Falls, Prevention, Medication therapy management, Physical therapy, Pharmacist, Pilot, Randomized controlled trial

\footnotetext{
* Correspondence: elizabeth_goldberg@brown.edu

'Department of Emergency Medicine, Brown University, 55 Claverick Street,

Providence, RI 02903, USA

2Department of Health Services, Practice and Policy, Brown University, $121 \mathrm{~S}$.

Main Street, Providence, RI 02912, USA

Full list of author information is available at the end of the article
}

(c) The Author(s). 2019 Open Access This article is distributed under the terms of the Creative Commons Attribution 4.0 International License (http://creativecommons.org/licenses/by/4.0/), which permits unrestricted use, distribution, and reproduction in any medium, provided you give appropriate credit to the original author(s) and the source, provide a link to the Creative Commons license, and indicate if changes were made. The Creative Commons Public Domain Dedication waiver (http://creativecommons.org/publicdomain/zero/1.0/) applies to the data made available in this article, unless otherwise stated. 


\section{Background}

The Centers for Disease Control and Prevention estimate that by 2030, nearly one in five persons in the US will be $\geq 65$ years old [1]. These older adults will comprise an increasing proportion of emergency department (ED) patients, and falls are the leading cause of fatal and non-fatal injuries that bring this population to the ED for evaluations [2]. ED evaluations for falls typically involve a focused assessment to rule out acute injuries, but do not identify modifiable risk factors to prevent subsequent falls [3-5]. ED patients rarely receive instructions to follow-up with clinicians that can address reasons for the fall [6]. Currently, only $3.7 \%$ of older adults receive fall guideline concordant care when they present to the ED after a fall [7]. According to the American Geriatrics Society and British Geriatrics Society guidelines, this care should include a multifactorial fall risk assessment that should be performed for all older persons who live at home and seek medical care in the ED because of a fall [8].

An interdisciplinary team of pharmacists and physical therapists (PTs) who perform standardized fall assessments is a key criterion for US geriatric ED accreditation [9]; however, currently, no applicable model exists to guide this care. Although falls are considered one of four high-yield research opportunities in geriatric emergency medicine [10], there is a notable lack of research in EDbased interventions to reduce the occurrence of recurrent falls among seniors. Preventing subsequent falls is critical to stop the cascade of functional decline, loss of independence, hospitalization, and death, which frequently follows a fall. Annual Medicare costs for adult falls are currently estimated to be $\$ 31.3$ billion [11], and failure to find new, effective strategies to address falls in this population will lead to increased costs as the US population ages.

To address the lack of fall prevention research in the ED, we developed GAPcare (the Geriatric Acute and Post-acute Fall Prevention Intervention). This intervention brings together patients, caregivers, pharmacy and PT professionals, and clinicians to provide a patient-centric, collaborative approach to fall prevention that bridges the ED visit with outpatient resources. Performing a fall prevention intervention in the ED (as opposed to after the ED visit) provides more timely evaluation, which is of critical importance because seniors are at high risk of recurrent falls in the immediate post-fall period [12]. Unlike other current fall prevention protocols, this intervention starts immediately after the fall in the ED when patients and caregivers are highly engaged and motivated to prevent further fall occurrences [13]. The purpose of this manuscript is to describe the study protocol of the initial GAPcare investigation.

\section{Purpose and methods}

The GAPcare study aims are to (1) examine the feasibility of the GAPcare intervention by assessing the number of participants who are eligible, recruited, and retained and measure the impact on ED length of stay (LOS); (2) determine if the GAPcare intervention (versus usual care) reduces subsequent falls and ED visits and hospital admissions in the 6-month follow-up; and (3) solicit feedback from participants on the utility and barriers of the GAPcare intervention and collect suggestions for improvement of the intervention. The rationale for completing this pilot trial prior to completing a definitive study powered for efficacy was to assess whether patients, caregivers, and clinicians were open to exploring prevention efforts with our pharmacists and PTs immediately after an injury, whether we could perform the intervention within the time constraints of a typical ED visit, and to obtain initial estimates of efficacy to help us determine the sample size for the subsequent definitive trial.

\section{Design of the GAPcare intervention and study protocol}

This study is a two-site parallel group, single-blinded pilot randomized controlled trial (RCT). The hospital Institutional Review Board approved the study. We registered the trial at ClinicalTrials.gov (NCT03360305). The GAPcare intervention incorporates key elements of prior fall interventions [14-18]. The intervention was developed from these key elements and shaped by expert opinion from a team of geriatricians, health service researchers, geriatric-specialized PTs, and case managers, as well as an emergency medicine residency-trained pharmacist. This group of experts also comprise this pilot RCT's research team and assisted in the creation of the study protocol.

\section{Setting}

The study is being conducted at two main sites that belong to the same health system in Providence, Rhode Island: Rhode Island Hospital, an academic tertiary-care hospital, and The Miriam Hospital, an academic community hospital. The two participating study sites represent the ideal environment for this pilot study. Rhode Island Hospital is the only federally designated Level I Trauma Center in the state and has an annual ED census of 105, 000. The Miriam Hospital provides care to a primarily community-dwelling geriatric population with a high injury and illness acuity and has an annual ED census of 73,000 .

\section{Eligibility}

Patients, their caregivers, and ED clinicians are recruited jointly to participate in the study. Study eligible patients are 65 years old or older, English-speaking, able to provide written informed consent (or have a legally authorized 
representative consent), and present to the ED after a fall. Participants must be community-dwelling or live in assisted or independent living communities. The treating clinician must intend to discharge the patient after their initial evaluation. Patients are excluded if their mental status is altered (i.e., intoxicated, agitated), they plan to leave the state in the ensuing 6 months (which limits retention for follow-up and engagement in treatment plans), are undomiciled, or cannot be reached by telephone.

\section{Recruitment and enrollment}

Research staff review the ED Electronic Health Record (EHR) $\left(\right.$ Epic $\left.^{\circ}\right)$ for ED patients presenting after a fall. Participants are recruited when PT and pharmacy specialists are available, Monday through Friday from 7 AM to 4 PM. The patient's ED clinician is asked to confirm that he/she plans to discharge the patient home. If this outcome is likely, the research staff confirms eligibility with the patient, invites the patient to enroll, and asks them to provide written consent for participation. Caregivers and treating clinicians are also asked to consent to study participation.

We first assess each patient's decisional capacity. For patients who score less than 4 on the Six-Item Screener [19] (a screen for cognitive impairment) but are interested in participating in the study, the legally authorized representative is asked to provide written consent.

After consent, each participant is randomized 1:1 to the intervention arm or usual care. Randomization is performed using REDcap and is based on randomization tables that stratify patients by study site. Enrollment staff do not have access to the allocation scheme before enrolling participants. It is not possible to blind the participants and treating clinicians to the allocation arm as they would be able to identify pharmacists and PTs at the bedside. However, the staff member who performs the assessment at 6-month follow-up is blinded to allocation arm.

\section{In-ED procedures}

After initial evaluation by the ED treatment team, patient participants are asked questions from the baseline questionnaire and their current medications are recorded by the research staff using previously published best practices for medication reconciliation [20]. Intervention patients receive individually tailored pharmacy and PT consultations according to the GAPcare protocol (see Table 1). Intervention participants receive all components of the intervention arm unless they refuse. The research staff delivers the intervention while participants are awaiting results of laboratory testing and imaging (see Fig. 1). Usual care participants receive care as dictated by the ED treatment team alone and a brochure from the Centers for Disease Control and Prevention about home safety. The research staff completes a timed up and go test on all participants regardless of study arm. All participants are provided with a fall calendar that allows them to record any new falls and healthcare visits at the time they occur for the following 6 months. It is at the discretion of the ED treatment team to contact the primary care physician (PCP), consult a case manager, and provide medical equipment (e.g., walkers, canes). Both intervention and usual care participants, their caregivers, and clinicians are asked to complete an end-of-visit survey to record their satisfaction with the care delivery, perceived barriers and facilitators, and suggestions for improvement.

\section{Follow-up procedures}

All participants receive telephone calls every 2 weeks to prompt them to complete fall calendars and provide them an opportunity to ask questions about study procedures

Table 1 In-ED procedures for intervention patients

\begin{tabular}{|c|c|c|}
\hline Personnel & Description & Administration \\
\hline \multicolumn{3}{|c|}{ Pharmacist assessment and medication-related action plan } \\
\hline Pharmacist & $\begin{array}{l}\text { The pharmacist study protocol consists of the following steps: } \\
\text { - Review the research staff obtained medication list [20]. } \\
\text { - Perform motivational interviewing with the patient and/or caregiver to identify 1-3 medication } \\
\text { recommendations, such as cessation or tapering of medication that increase fall risk. } \\
\text { - Communicate the medication-related action plan (MRAP) in writing to the patient and ED treatment team. A } \\
\text { facsimile copy of the MRAP is sent to the primary care provider (PCP) at the end of the visit via the newly } \\
\text { created EHR structure. }\end{array}$ & $\begin{array}{l}\cdot 20 \text { min } \\
\text { - In-person } \\
\text { evaluation }\end{array}$ \\
\hline \multicolumn{3}{|c|}{ Physical therapy assessment and action plan } \\
\hline Physical therapist & $\begin{array}{l}\text { The PT has the following responsibilities: } \\
\text { - Performs a gait, balance, and lower extremity strength assessment. } \\
\text { - Assesses the patient's ability to function independently on discharge and assists with discharge planning. } \\
\text { - Recommends outpatient services/referrals, such as referral to outpatient or home PT and occupational therapy, a } \\
\text { home-safety evaluation, community fall prevention programs, or if necessary direct admission to a skilled nursing } \\
\text { facility. } \\
\text { - Communicates the PT action plan (PTAP) in writing and in person to the patient and ED treatment team. A } \\
\text { facsimile copy of the PTAP is sent to the PCP at the conclusion of the visit via the newly created EHR structure. }\end{array}$ & $\begin{array}{l}\cdot 20-30 \text { min } \\
\cdot \text { In-person } \\
\text { evaluation }\end{array}$ \\
\hline
\end{tabular}




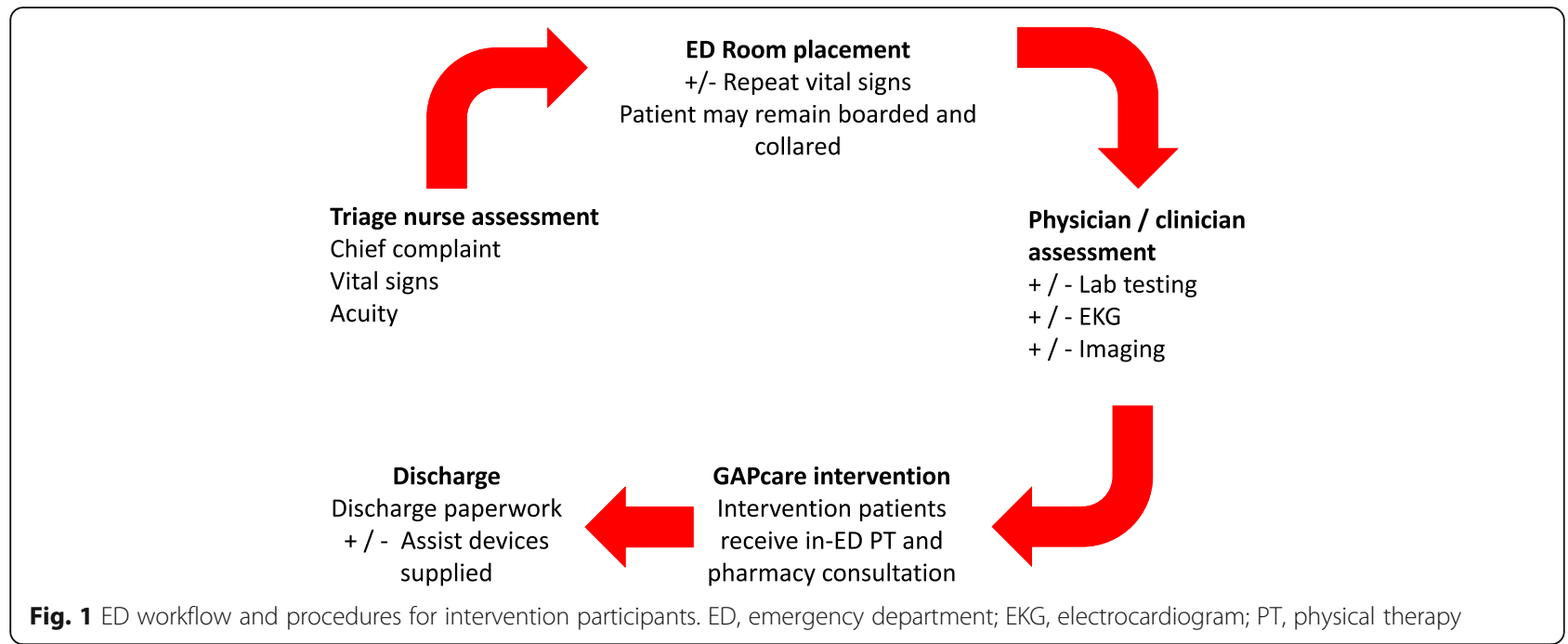

(Fig. 2). At 30 and 90 days after enrollment, participants complete a follow-up survey over the telephone, via email, or in person, depending on participant preference. At 180 days after enrollment, research staff, who are blinded to arm assignment, conduct a home visit with participants to perform the final procedures and assessments (Table 2). Fall calendars are collected at the conclusion of the visit. Research staff masked to arm allocation also abstract study outcomes from the EHR to confirm and supplement the data recorded in the fall calendars.

\section{Efforts to improve recruitment and retention}

A number of steps are taken to enhance recruitment and retention of participants. First, an Epic ${ }^{\circ}$ Best Practice Advisory alerts research staff that a potentially eligible study subject is in the ED. Second, patients are asked during their index visit how they prefer to be contacted (by telephone or e-mail) and their preferred time of day for follow-up communication. Research staff schedule future follow-up telephone calls with patients during the 2-week reminder calls. If patients cannot be reached by telephone by the research staff, the principal investigator (PI) will attempt to contact them. Finally, an in-person visit is conducted for participants who cannot be contacted.

\section{Data collection}

All participants receive an in-ED assessment by the research staff and scheduled follow-ups at 30, 90, and 180 days. Caregiver and clinician interviews occur only

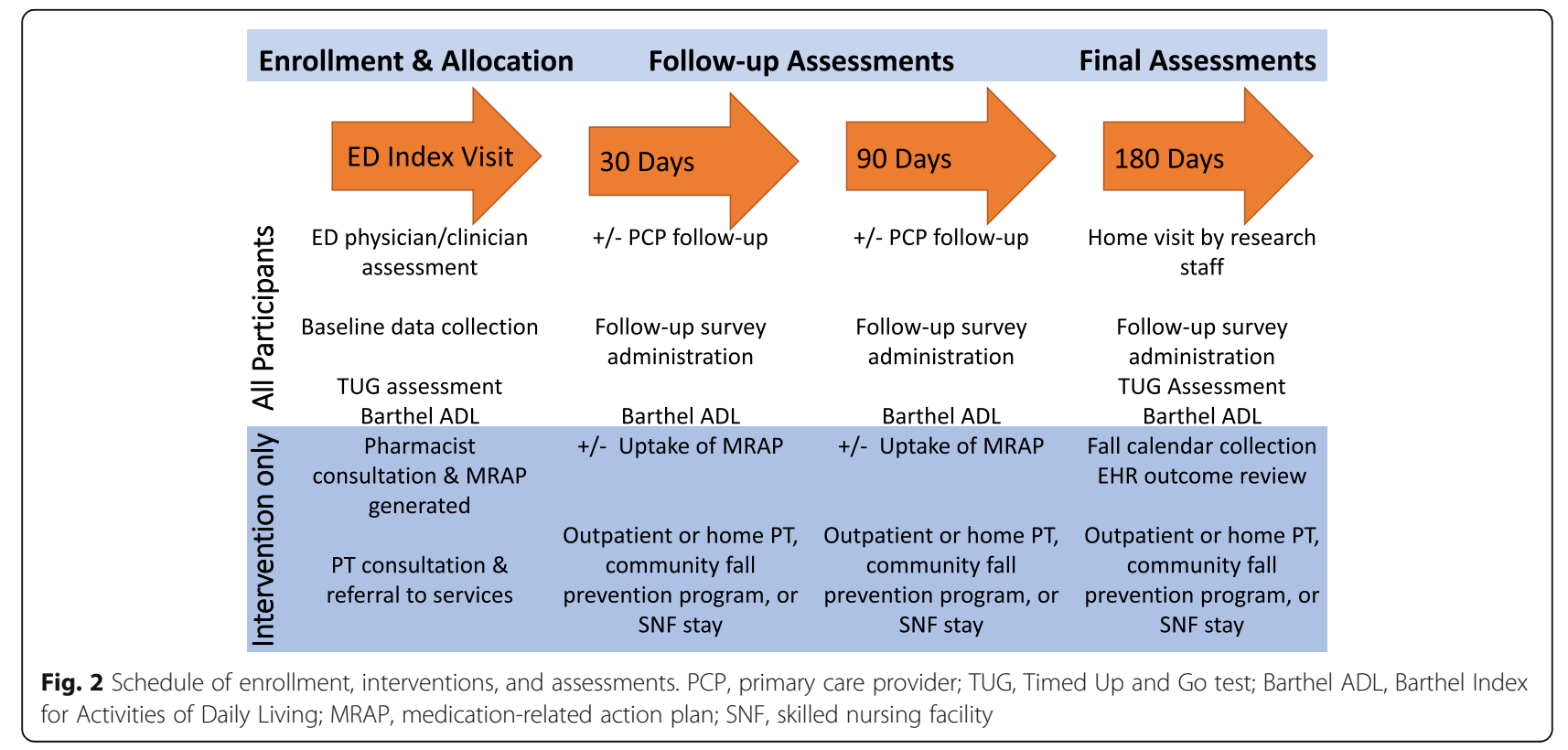


Table 2 Study instruments and timeline of assessments

\begin{tabular}{|c|c|c|}
\hline Instrument & Description & Administration \\
\hline \multicolumn{3}{|l|}{ Process evaluation } \\
\hline $\begin{array}{l}\text { Screening, eligibility, and } \\
\text { retention }\end{array}$ & $\begin{array}{l}\text { - Records how many patients were screened, agreed to participate, were recruited, received } \\
\text { intended treatment, and were retained }\end{array}$ & $\begin{array}{l}\text { - Baseline, } 180 \\
\text { days }\end{array}$ \\
\hline ED component and fidelity & $\begin{array}{l}\text { - Records index visit ED LOS, time between consult call and arrival of pharmacist and PT, } \\
\text { length of each consult, accuracy and completeness of action plan compared to the protocol }\end{array}$ & - Baseline \\
\hline $\begin{array}{l}\text { Patient, caregiver, and clinician } \\
\text { feedback }\end{array}$ & $\begin{array}{l}\text { - Tracks satisfaction with each component of the evaluation, perceived barriers and facilitators, } \\
\text { and collects suggestions for improvement of the intervention }\end{array}$ & - Baseline \\
\hline Follow-up component & $\begin{array}{l}\text { - Tracks follow-up phone and in-person sessions with the research staff, PT, and any home } \\
\text { services. Records uptake of recommendations made on the action plan for intervention } \\
\text { participants }\end{array}$ & $\begin{array}{l}\cdot 30,90,180 \\
\text { days }\end{array}$ \\
\hline \multicolumn{3}{|c|}{ Outcome evaluation-feasibility, fidelity, and initial efficacy } \\
\hline Enrollment questionnaire & $\begin{array}{l}\text { - Demographic characteristics (includes marital status, whether the patient lives alone or with } \\
\text { others, educational attainment, current employment.) } \\
\text { - Prior fall history, comorbidities, ED index visit fall circumstances, and injuries }\end{array}$ & $\begin{array}{l}\cdot \text { Baseline } \\
\cdot<5 \text { min }\end{array}$ \\
\hline Six-Item Screener (SIS) [19] & $\begin{array}{l}\text { - 6-point questionnaire to measure cognitive impairment for study screening [19], }<4 \text { indicates } \\
\text { high risk for cognitive impairment }\end{array}$ & $\begin{array}{l}\cdot \text { Baseline } \\
\cdot 2 \text { min }\end{array}$ \\
\hline $\begin{array}{l}\text { Timed Up and Go (TUG) test } \\
\text { [21] }\end{array}$ & $\begin{array}{l}\text { - Timed test of how long it takes the patient to get up from a chair, walk } 10 \text { feet, and return to } \\
\text { the chair } \\
\text { - Validated measure of current function, balance, and fall risk } \\
\text { - A TUG }>12 \text { seconds identifies those patients at greater risk for subsequent falls }\end{array}$ & $\begin{array}{l}\cdot \text { Baseline, } 180 \\
\text { days } \\
\cdot<5 \text { min }\end{array}$ \\
\hline $\begin{array}{l}\text { Barthel Index for Activities of } \\
\text { Daily Living (ADL) [22] }\end{array}$ & $\begin{array}{l}\text { - Used to assess functional independence [22] } \\
\text { - Information can be gained by self-report or caregiver report }\end{array}$ & $\begin{array}{r}\cdot \text { Baseline, } 30, \\
90,180 \text { days }\end{array}$ \\
\hline Tinetti [23] & $\begin{array}{l}\text { - Validated measure of gait and balance } \\
\text { - Predicts falls }\end{array}$ & $\begin{array}{l}\cdot \text { Baseline } \\
\cdot<5 \text { min }\end{array}$ \\
\hline 5 Times Sit to Stand [24] & - A measure of functional lower limb muscle strength & - Baseline \\
\hline AM-PAC "6-clicks" & $\begin{array}{l}\text { - Test of activity limitations and function [25] used to help determine need and intensity of } \\
\text { outpatient services such as PT, safety of discharge, and to predict falls and healthcare } \\
\text { utilization }\end{array}$ & $\begin{array}{l}\cdot \text { Baseline } \\
\cdot 1 \mathrm{~min}\end{array}$ \\
\hline $\begin{array}{l}\text { Falls Efficacy Scale-International } \\
{[26]}\end{array}$ & - Measures fear of falling, which increases fall risk & - 180 days \\
\hline Medication questionnaire & $\begin{array}{l}\text { - Records all current and new prescriptions, over-the-counter and herbal medication, } \\
\text { recommendations made by the pharmacist, recommendations accepted by the patient, and } \\
\text { date of initiation/cessation }\end{array}$ & $\begin{array}{l}\cdot \text { Baseline, } 180 \\
\text { days } \\
\cdot 25 \text { min }\end{array}$ \\
\hline Outcome instrument & $\begin{array}{l}\text { - Records follow-up falls and injuries by self-report and EHR review } \\
\text { - Records subsequent ED visits and hospitalizations }\end{array}$ & $\begin{array}{l}\cdot 30,90,180 \\
\text { days } \\
.5 \text { min }\end{array}$ \\
\hline
\end{tabular}

ED emergency department, LOS length of stay, SIS Six-Item Screener, TUG Timed Up and Go, ADL Activities of Daily Living, PT physical therapy, AM-PAC Activity Measure for Post-Acute Care, EHR electronic health record

during the index ED visit. The information that is collected from participants and the EHR is summarized in Table 2.

We ensure fidelity to the research protocol through several means. First, the study coordinator reviews baseline enrollment data on a weekly basis. Second, the study team reviews on a monthly basis the proportion of subjects who are eligible, approached, consent to participate, and enrolled. Reasons for refusal are tracked to allow the study team to modify how they communicate study details when they approach the participant and for subsequent study planning. The PI holds weekly meetings with the research staff to discuss study progress and address concerns. Finally, the biostatistician performs automated and manual checks in the REDcap data collection program to ensure data quality.

\section{Sample size}

This early stage investigation is designed to provide a preliminary indication of the proportion of screened participants who are eligible, recruited, and retained, with the goal of estimating these proportions with a standard error of less than $5 \%$. Assuming the maximum possible variability for each of these proportions, the sample size of 120 produces a standard error of $4.6 \%$, enabling estimation of a margin of error of $\pm 8.9 \%$. A second feasibility aim was estimating if there was a major difference in ED LOS between groups. For $\alpha=0.05$ with a two-sided 
test and assuming a standard deviation of $120 \mathrm{~min}$, a sample size of 52 per group has adequate power $(\beta=$ 0.83 ) to detect a difference of $65 \mathrm{~min}$. or more. To allow for non-parametric tests which may be necessary given the skewed nature of ED visits, a $15 \%$ increase in sample size or 60 per group will yield the same power [27].

\section{Data analysis \\ Feasibility}

We collect the following feasibility measures and will report them descriptively [28]: number of patients screened, proportion eligible, number of patients recruited, time required to recruit, number of patients unable to provide consent, number of patient refusals, number of dropouts, and retention at each follow-up time point. We will use frequencies, proportions, rates, means/medians, and standard deviations and other measures of variability, as appropriate, to report on these feasibility measures. Also, we keep a record of lessons learned during the implementation of this study. To compare the median ED LOS between participants in both study arms, we will use the Wilcoxon-Mann-Whitney test and bootstrapping to generate a 95\% confidence interval for the median difference in ED LOS. We will also examine the length of time required to complete the pharmacy and PT consultation in the intervention arm to determine if the intervention is feasible within the timeframe of an ED visit.

\section{Fidelity}

We will use descriptive statistics to assess key parameters of fidelity: receipt of the pharmacy and PT consultations, MRAP provision to patients and receipt of the MRAP by PCPs, fall calendar completion, and uptake of recommendations by patients and PCPs at 6-month follow-up. Implementation activities will be measured against recommendations and categorized into three level of adherence: adherent, partially adherent, and non-adherent.

\section{Initial efficacy}

We will complete the analyses on an intention-to-treat basis; participant outcomes will be analyzed according to their allocated arm, irrespective of the intervention received. Therefore, patients who are admitted or discharged to a skilled nursing facility after randomization will be included in the analysis.

We will compare the proportion and median/mean number of recurrent falls, ED visits, and hospital admissions over 6 months occurring in each arm using Fisher's exact test (proportions) and Wilcoxon's and Student's $t$ test (occurrence). If sample size permits, we will conduct a survival analysis for a time-to-event analysis. Survival time will be defined as the time from randomization to the time of the first recurrent fall. Dates and times for this outcome will be obtained from the fall calendar and EHR review. For this analysis, we will first use the Kaplan-Meier method to estimate time to the recurrent fall. Next, we will use the log-rank test to test differences between survival curves for the intervention vs. usual care arm. Finally, hazard ratios and $95 \%$ confidence intervals will be calculated using the Cox proportional hazards model; additional analyses will adjust for baseline differences in physical function. We may also use multivariate frailty models, which are an extension of traditional survival analyses that allow for multiple failures (in this case falls) and include random effects that take into account underlying individual predisposition to falling.

\section{Data and safety monitoring plan}

Potential study-related adverse events or unintended effects will be reported to the data monitoring committee. This committee is composed of researchers who have no direct involvement in the study or direct relationship with the sponsor. The PI is ultimately responsible for ensuring participant safety throughout the trial period. An interim analysis is not planned. Protocol modifications will be reported to the Institutional Review Board.

\section{Discussion}

GAPcare is a two-hospital, single-blind, randomized controlled trial that examines the feasibility of delivering an in-ED multicomponent fall prevention intervention with coordination of post-discharge services to communitydwelling older adults. This initiative is the first US trial to randomly assign patients who present with falls to a pharmacy and PT evaluation while they await routine care in the ED. This study is innovative for several reasons. Prior fall prevention studies have excluded patients with cognitive impairment and Parkinson's disease, or those near the end of life. GAPcare includes patients regardless of dementia status, chronic disease burden, or prognosis. Therefore, our study results will be applicable to seniors who present to other academic medical centers. Although ED clinicians benefit from the team-based approach and expertise of the pharmacist and PT consults, ED clinicians are not called on to administer assessments or change their current ED workflow. This design allows for implementation of the study protocol without increasing clinician burden or requiring intensive pre-implementation education of ED physicians and staff.

The ED visit after a fall represents a "teachable moment" where patients are likely to recognize the need for prevention of future falls. Caregivers are often present and maximally engaged during this ED evaluation, as their loved one has just experienced an injury. The potential benefit of fall prevention is apparent to both the patient and the caregiver. Thus, we believe that this model addresses barriers to effective fall interventions identified in prior qualitative studies such as the denial of falling risk, the belief that fall prevention is not necessary, and practical impediments to attendance at follow-up appointments 
[29]. Because the intervention takes place in the ED, participants do not need to arrange transportation to complete the study. In addition, the immediate post-fall period is a high-risk time for older adults to have repeat falls [12]. This intervention is delivered prior to patients returning home, which is an ideal time to prevent falls in this high-risk period.

There are two main limitations of this study. First, this study is not designed or powered to measure a reduction in recurrent falls. A subsequent efficacy study will be performed with adequate power to test this aim. Second, both study sites are in a mid-sized urban area and the study results will not be generalizable to EDs that are in rural areas or EDs without availability of in-ED pharmacist or PT consultation. However, we anticipate that EDs with geriatric ED accreditation may use this model of care for their patients.

In the past decade, deaths from falls have increased by $30 \%$ and falls remain the leading cause of injury-related deaths in older Americans [30]. Falls are common, often preventable, and have a significant cost to the individual and society. The ED is ideally positioned to serve as a screening site for falls and to start prevention efforts. GAPcare pilots a new model of care that could prevent falls in the high-risk patient population that requires emergency care after a fall.

\section{Abbreviations}

AM-PAC: Activity Measure for Post-Acute Care; Barthel ADL: Barthel Index for Activities of Daily Living; ED: Emergency department; EHR: Electronic health record; EKG: Electrocardiogram; GAPcare: The Geriatric Acute and Post-acute Fall Prevention Intervention; LOS: Length of stay; MRAP: Medication-related action plan; PCP: Primary care provider; PI: Principal investigator; PT: Physical therapist; PTAP: Physical therapy action plan; SIS: Six-Item Screener; SNF: Skilled nursing facility; TUG: Timed Up and Go

\section{Acknowledgements}

Not applicable

\section{Authors' contributions}

EG conceived, drafted, and revised the manuscript. LR, SM, and RM made substantial contributions to the conception and design of the study, as well as revised the manuscript. All authors have approved the final manuscript to be published.

\section{Funding}

Research support was provided by the following sponsors: National Institute on Aging (R03AG056349), SAEMF/EMF GEMSSTAR for Emergency Medicine Supplemental Funding (RF2017-010), and Agency for Healthcare Research and Quality (T32 HS000011-30). These grants were awarded after undergoing peer review by the National Institutes of Health. The content is solely the responsibility of the authors and does not reflect the official views of the National Institutes of Health.

\section{Availability of data and materials}

Human subject protections preclude us from granting public access to participant-level data. However, aggregate results will be published in a peer-reviewed journal after study completion. Access to the full protocol and statistical code will be available after study completion.

\section{Ethics approval and consent to participate}

The hospital Institutional Review Board approved the study. We registered the trial at clinicaltrials.gov (NCT03360305). It is viewable at https://
clinicaltrials.gov/ct2/show/NCT03360305. Trial registration date: December 4, 2017. All participants provided consent for participation in the study. Those subjects without decisional capacity must have a legally authorized representative present who provides written informed consent with assent from the patient.

\section{Consent for publication}

Not applicable

\section{Competing interests}

The authors declare that they have no competing interests.

\section{Author details}

'Department of Emergency Medicine, Brown University, 55 Claverick Street, Providence, RI 02903, USA. 'Department of Health Services, Practice and Policy, Brown University, 121 S. Main Street, Providence, RI 02912, USA. ${ }^{3}$ Providence VA Medical Center, 830 Chalkstone Ave, Providence, RI 02908, USA. ${ }^{4}$ Department of Emergency Medicine, Brigham and Women's Hospital, Harvard University, 75 Francis Street, Boston, MA 02115, USA.

Received: 7 December 2018 Accepted: 14 August 2019

Published online: 27 August 2019

\section{References}

1. Albert M, Rui P, McCaig LF. Emergency department visits for injury and illness among adults aged 65 and over: United States, 2012-2013. NCHS Data Brief. 2017;(272):1-8.

2. Bergen $G S M$, Burns ER. Falls and fall injuries among adults aged $\geq 65$ years — United States, 20142016 [cited 2016 September 23, 2016]. 65:[Available from: https://www.cdc.gov/mmwr/volumes/65/wr/mm6537a2.htm?s_cid= mm6537a2_w.

3. Davies AJ, Kenny RA. Falls presenting to the accident and emergency department: types of presentation and risk factor profile. Age Ageing. 1996; 25(5):362-6.

4. Khan SA, Miskelly FG, Platt JS, Bhattachryya BK. Missed diagnoses among elderly patients discharged from an accident and emergency department. J Accid Emerg Med. 1996;13(4):256-7.

5. Carpenter CR, Griffey RT, Stark S, Coopersmith CM, Gage BF. Physician and nurse acceptance of technicians to screen for geriatric syndromes in the emergency department. West J Emerg Med. 2011;12(4):489-95.

6. Paniagua MA, Malphurs JE, Phelan EA. Older patients presenting to a county hospital ED after a fall: missed opportunities for prevention. Am J Emerg Med. 2006;24(4):413-7.

7. Salter AE, Khan KM, Donaldson MG, Davis JC, Buchanan J, Abu-Laban RB, et al. Community-dwelling seniors who present to the emergency department with a fall do not receive guideline care and their fall risk profile worsens significantly: a 6-month prospective study. Osteoporos Int. 2006;17(5):67283.

8. The American Geriatrics Society (AGS) and British Geriatrics Society (BGS) Panel on the Clinical Practice Guideline for the Prevention of Falls in Older Persons. Prevention of Falls in Older Persons: AGS/BGS Clinical Practice Guideline. 2010.

9. Carpenter $\mathrm{CR}$, Hwang U, Biese $\mathrm{K}$, Carter D, HOgan T, Karounos M, Malone M, Melady D, Rosen A, Rosenberg M, Schneider S, Shah M, Spiegel T, Stern M. ACEP accredits geriatric emergency care for emergency departments ACEPNow 2017 [Available from: http://www.acepnow.com/article/acepaccredits-geriatric-emergency-care-emergency-departments/.

10. Carpenter CR, Shah MN, Hustey FM, Heard K, Gerson LW, Miller DK. High yield research opportunities in geriatric emergency medicine: prehospital care, delirium, adverse drug events, and falls. J Gerontol A Biol Sci Med Sci. 2011;66(7):775-83

11. Burns ER, Stevens JA, Lee R. The direct costs of fatal and non-fatal falls among older adults - United States. J Safety Res. 2016;58:99-103.

12. Lam C, Kang J-H, Lin H-Y, Huang H-C, Wu C-C, Chen P-L. First fall-related injuries requiring hospitalization increase the risk of recurrent injurious falls: a nationwide cohort study in Taiwan. PLoS ONE. 2016;11(2):e0149887.

13. National Institutes of Health. $\mathrm{NHH}, \mathrm{PCORI}$ announce major award to prevent falls injuries in older people 2014 [Available from: https:/www.nih.gov/ news-events/news-releases/nih-pcori-announce-major-award-prevent-fallsinjuries-older-people. 
14. Training Modules for Fall Prevention: The STRIDE Study 2016 [Available from: http://www.stride-study.org/providers/training/.

15. Stevens JA. The STEADI tool kit: a fall prevention resource for health care providers. IHS Prim Care Provid. 2013;39(9):162-6.

16. Baraff $\sqcup$, Della Penna R, Williams N, Sanders A. Practice guideline for the ED management of falls in community-dwelling elderly persons. Kaiser Permanente Medical Group. Ann Emerg Med. 1997;30(4):480-92.

17. Caplan GA, Williams AJ, Daly B, Abraham K. A randomized, controlled trial of comprehensive geriatric assessment and multidisciplinary intervention after discharge of elderly from the emergency department--the DEED II study. J Am Geriatr Soc. 2004;52(9):1417-23.

18. Close J, Ellis M, Hooper R, Glucksman E, Jackson S, Swift C. Prevention of falls in the elderly trial (PROFET): a randomised controlled trial. Lancet. 1999; 353:93-7.

19. Carpenter CR, DesPain B Fau-Keeling TN, Keeling Tn Fau-Shah M, Shah M Fau-Rothenberger M, Rothenberger M. The Six-Item Screener and AD8 for the detection of cognitive impairment in geriatric emergency department patients. 2011 (1097-6760 (Electronic)).

20. Pippins JR, Gandhi TK, Hamann C, Ndumele CD, Labonville SA, Diedrichsen EK, et al. Classifying and predicting errors of inpatient medication reconciliation. J Gen Intern Med. 2008;23(9):1414-22.

21. Podsiadlo D, Richardson $S$. The timed "Up \& Go": a test of basic functional mobility for frail elderly persons. J Am Geriatr Soc. 1991;39(2):142-8.

22. Mahoney Fl, Barthel DW. Functional evaluation: the Barthel index. Md State Med J. 1965;14:61-5.

23. Tinetti ME, Williams TF, Mayewski R. Fall risk index for elderly patients based on number of chronic disabilities. Am J Med. 1986;80(3):429-34.

24. Whitney SL, Wrisley DM, Marchetti GF, Gee MA, Redfern MS, Furman JM. Clinical measurement of sit-to-stand performance in people with balance disorders: validity of data for the Five-Times-Sit-to-Stand Test. Phys Ther. 2005;85(10):1034-45.

25. Jette DU, Stilphen M, Ranganathan VK, Passek SD, Frost FS, Jette AM. Validity of the AM-PAC "6-Clicks" inpatient daily activity and basic mobility short forms. Physical therapy. 2014;94(3):379-91.

26. Yardley L, Beyer N, Hauer K, Kempen G, Piot-Ziegler C, Todd C. Development and initial validation of the Falls Efficacy Scale-International (FES-I). Age Ageing. 2005:34(6):614-9.

27. Erich L. Lehmann. Nonparametrics: statistical methods based on ranks, Revised 1998. 76-81 p.

28. Lancaster GA, Dodd S, Williamson PR. Design and analysis of pilot studies: recommendations for good practice. J Eval Clin Pract. 2004;10(2):307-12.

29. Yardley L, Bishop FL, Beyer N, Hauer K, Kempen Gl, Piot-Ziegler C, et al. Older people's views of falls-prevention interventions in six European countries. Gerontologist. 2006;46(5):650-60.

30. Burns $E$, Kakara R. Deaths from falls among persons aged $\geq 65$ years United States, 2007-2016. MMWR Morb Mortal Wkly Rep. 2018;67:509-14.

\section{Publisher's Note}

Springer Nature remains neutral with regard to jurisdictional claims in published maps and institutional affiliations.

Ready to submit your research? Choose BMC and benefit from:

- fast, convenient online submission

- thorough peer review by experienced researchers in your field

- rapid publication on acceptance

- support for research data, including large and complex data types

- gold Open Access which fosters wider collaboration and increased citations

- maximum visibility for your research: over $100 \mathrm{M}$ website views per year

At $\mathrm{BMC}$, research is always in progress.

Learn more biomedcentral.com/submissions 